\title{
A retrospective analysis of urine culture results issued by the microbiology department, Teaching Hospital, Karapitiya
}

\author{
LU Edirisinghe ${ }^{1}$, D Vidanagama ${ }^{2}$ \\ ${ }^{1}$ Senior Registrar in Medicine, ${ }^{2}$ Consultant Microbiologist, Teaching Hospital, Karapitiya, Galle.
}

\begin{abstract}
Objectives: To find out the common pathogenic organisms causing urinary tract infection in patients admitted to the Teaching Hospital, Karapitiya and to review the antibiotic sensitivity pattern of these organisms by analyzing the urine culture results generated by the microbiology laboratory.

Design: All the urine culture and antibiotic sensitivity test (ABST)) reports of patients over the age of 12 years generated by the microbiology laboratory between January to May 2008 were collected and analyzed.
\end{abstract}

Setting: The Teaching hospital, Karapitiya.

Materials: We have analyzed 1772 urine culture and ABST reports generated between January and May, 2008.

Results: This study revealed that coliforms were the predominant pathogenic organisms isolated from the urine samples which were sent for culture and ABST from January to May 2008 and there was emerging resistance to many antibiotics including the commonly used quinolones such as nalidixic acid and norfloxacin.

\section{Background}

Urinary tract infections are one of the commonest infections that we encounter in hospital practice. They are usually classified according to the anatomical part of the urinary tract affected; kidneys (acute pyelonephritis), bladder (cystitis), urethra (urethritis). It can be either community acquired or hospital acquired. The common aetiological agents are Escherichia coli, Pseudomonas spp., Proteus spp., Klebsiella spp. and Enterococcus spp. ${ }^{1}$.

A simple urinary analysis (urine full report) is a good screening test but in order to confirm the diagnosis and to find out the causative organism a urine culture is required.

Widespread use of antibiotics without properly identifying the organism or its antibiotic sensitivity pattern has led to recurrent urinary tract infections or the emergence of multi-drug resistant organisms. One emerging threat is multi-drug resistant E.coli clonal group ${ }^{2}$.

An Indian study had shown that out of 100 culture positive urine samples, $42 \%$ of the isolates were detected to produce extended spectrum beta lactamase (ESBL) among which $34.42 \%$ were E.coli isolates ${ }^{3}$. These isolates were resistant to the commonly used antibiotics.

Another study conducted in Kuwait also showed a relatively high prevalence of clinically significant ESBL producers among the Enterobacteriaceae and Pseudomonas spp. in their hospitals. It also revealed that the most common infections associated with ESBLproducing pathogens were urinary tract infection $(68.2 \%)$, followed by wound infection $(14.4 \%)$ and bloodstream infection $(6.1 \%)^{4}$.

ESBL-producing bacteria may not be detected by routine disk diffusion susceptibility test. If special efforts are not made, this may lead to inappropriate use of antibiotics and treatment failure.

Another study conducted in Italy revealed that multi-resistant uropathogens showed a significant positive correlation with an indwelling bladder catheter and the age. An upward trend in the resistance of E.coli to cotrimoxazole, ampicillin and fluoroquinolones was observed from 1996 to 1999 where more 
than $50 \%$ of Pseudomonas strains were resistant to fluoroquinolones and gentamicin ${ }^{5}$.

In our hospital, only limited data is available regarding the prevalence of ESBL-producing bacteria. Even though it is well known that E.coli is the commonest pathogenic organism responsible for urinary tract infections, limited amount of data is available with regards to the prevalence of this organism and its sensitivity to the commonly used antibiotics among patients who are admitted to the Teaching Hospital, Karapitiya. Therefore, we have planned this study to explore the prevalence of the various pathogenic organisms in our hospital and further to find out their antibiotic sensitivity pattern.

\section{Current practice}

Whenever a patient presents with symptoms of urinary tract infection, a urine full report is requested and if there are pus cells, antibiotics are started with or without a urine culture and ABST being performed. The most commonly used antibiotics are oral Norfloxacin, Ciprofloxacin and Nalidixic acid. In the outpatient department as well as in the wards Nitrofurantoin and Nalidixic acid are freely available to treat patients with urinary tract infections. This is the situation in other state hospitals, too.

\section{Audit on isolated organisms and their sensitivity pattern}

All the urine culture and antibiotic sensitivity request forms send to the microbiology laboratory, Teaching Hospital, Karapitiya from January to May 2008 were collected and analyzed separately. The request forms in which the age was below 12 years were not counted. The culture results and sensitivity patterns are usually documented in the back of the request form itself. When a significant isolate (i.e. yielding $>10^{5} \mathrm{CFU} / \mathrm{mL}$ or $10^{4-5} \mathrm{CFU} / \mathrm{mL}$ of a pure growth of bacteria), was detected, they were counted as positive samples while scanty or insignificant growths and cultures with no growths were counted as negative samples. Mixed growths were considered as contaminants. In the positive samples antibiotic sensitivity pattern, patient's age, sex, co-morbid factors, catheterized or not were documented in a separate sheet.

\section{Results}

In this audit we have analyzed a total number of 1772 culture reports generated by the microbiology laboratory between January and May 2008. There was very high percentage of culture negative samples (69\%). Out of the culture positive samples, the commonest organisms isolated were coliforms (89\%). Other organisms such as Pseudomonas spp. (5\%), Enterococcus spp. (4.45\%) and Staphylococcus aureus $(1.4 \%)$ were relatively rare (Table 1 ). Out of the 320 coliforms isolated samples, 10 were found to be producing ESBL enzymes. In fact $47.5 \%$ of the coliforms isolated were resistant to norfloxacin which is commonly used to treat urinary tract infections in our hospital (Table 2). Further, the coliforms were highly resistant to ampicillin (80\%). All the Pseudomonas isolates were sensitive only to very potent antibiotics such as amikacin, imipenem, meropenem and netilmicin. In our study, the urinary tract infections caused by coliforms were almost equally distributed among the young, the middle aged and the elderly patients while Pseudomonas infections were predominantly seen among the elderly patients (Table 3). 
Table 1 - Number of organisms isolated in each month.

\begin{tabular}{lcccccc}
\hline \multicolumn{1}{c}{ Organism } & January & February & March & April & May & Total \\
\hline Coliforms & 59 & 74 & 43 & 59 & 85 & 320 \\
Pseudomonas spp. & 7 & 5 & 2 & & 4 & 18 \\
Enterococci. & 3 & 5 & 5 & & 3 & 16 \\
Staph Aureus & & 3 & & 2 & & 5 \\
No growth. & 235 & 286 & 160 & 203 & 340 & 1224 \\
Mixed growths. & 30 & 22 & 20 & 55 & 62 & 189 \\
\hline \multicolumn{1}{c}{ Total } & $\mathbf{3 3 4}$ & $\mathbf{3 9 5}$ & $\mathbf{2 3 0}$ & $\mathbf{3 1 9}$ & $\mathbf{4 9 4}$ & $\mathbf{1 7 7 2}$ \\
\hline
\end{tabular}

Table 2 - Number of coliforms isolated urine samples tested for common antibiotics and percentage of resistance samples identified.

\begin{tabular}{lccc}
\hline \multicolumn{1}{c}{ Drug } & $\begin{array}{c}\text { Tested } \\
\text { Samples }\end{array}$ & $\begin{array}{c}\text { Resistant } \\
\text { Samples }\end{array}$ & Percentage \\
\hline Nitrofurantoin & 291 & 91 & $31 \%$ \\
Co-amoxiclave & 276 & 118 & $43 \%$ \\
Norfloxacin & 307 & 146 & $47.5 \%$ \\
Co-trimaxasole & 263 & 129 & $51 \%$ \\
Nalidixic acid & 291 & 174 & $66.6 \%$ \\
Ampicillin & 230 & 182 & $80 \%$ \\
\hline
\end{tabular}

Table 3 - The percentage of coliforms and Pseudomonas spp. isolated in different age groups.

\begin{tabular}{ccc}
\hline Age group & Coliforms & Pseudomonas \\
\hline $12-40$ & $35 \%$ & $31.25 \%$ \\
$40-60$ & $23 \%$ & $18.75 \%$ \\
$60-90$ & $30 \%$ & $50 \%$ \\
\hline
\end{tabular}

\section{Discussion}

The increasing prevalence of infections caused by antibiotic-resistant bacteria makes the empirical treatment of urinary tract infections more difficult. One of the important factors contributing to these high resistance rates might be the increasing use of antibiotics without knowing the causative organism and its antibiotic sensitivity pattern. In this study, we found that coliforms were the commonest offending organism in the majority of urinary tract infections in patients who were admitted to Teaching Hospital, Karapitiya and it was almost evenly distributed among the young, the middle aged and the elderly patients. In contrast,
Pseudomonas spp. infections were predominantly identified among the elderly patients. Furthermore, the isolated Pseudomonas were extremely resistant to quinolones and sensitive to netilmicin, amikacin, imipenem or meropenem. Therefore, when treating elderly patients with urinary tract infections, it is very important to do cultures and if the isolate is a Pseudomonas spp., one has to keep in mind about its resistance to quinolones.

Apart from that, antibiotic resistance among the Coliforms is an emerging threat especially among hospitalized patients. In this hospital, it is now evident that there is a significant number of coliform isolates which were resistant to 
quinolones (Table 2). In fact, in ten urine samples they were identified as ESBL producers. Apart from quenolones, nitrofurantoin is also used as a first line drug in the treatment of urinary tract infections. Further, we identified that $31 \%$ of the coliforms were resistant to Nitrofurantoin. Therefore, it is evident that resistance among coliforms was lesser for nitrofurantoin than for norfloxacin (47.5\%) and nalidixic acid (66.6\%) (Table $2 \&$ Figure 1).

One Spanish study conducted in 2002 revealed that E.coli was the main uropathogen and the overall fluoroquinolone resistance was near $23 \% .^{6}$ But in our study population, this number is a little higher probably due to increased usage of this group of drugs to treat urinary tract infections. In fact, the past favourite, nitrofurantoin, which is at present rarely used as the first choice, is now emerging as more effective against coliforms than Nalidixic acid or quinolones.

A major drawback of this audit is the incompletely filled request forms and some forms not having information on at least the patient's age or sex. Those cases were classified according to the ward number. Only one request form mentioned that the patient is on a catheter and very few request forms gave a proper history and other co-morbid factors such as diabetes. Furthermore, all the urine samples were not tested for the commonly used oral antibiotics due to the limitation of discs. Therefore, we had to calculate the resistance according to the number of samples tested for that antibiotic (Table 2).

\section{Figure 1 - Drug resistance to Coliforms}

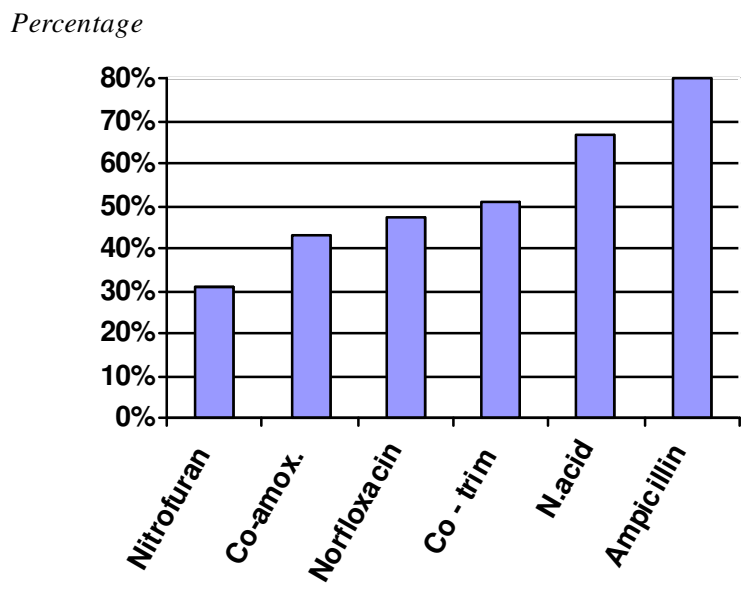

\section{Conclusions}

In this study we have identified the importance of completing the request forms in detail which will enable the microbiologist to identify high risk patients early as well as the resistant strains. Having done so, we have to repeat a similar audit later in order to identify any significant change in the above findings. It is also important to send urine for culture and ABST in patients suspected of having urinary tract infections before starting antibiotics due to the increased prevalence of resistance among pathogens to oral antibiotics that are frequently used to treat urinary tract infections.

The information generated in this kind of retrospective analysis may be useful for the clinicians to decide on empiric treatment in an era where highly antibiotic resistant bacterial strains are emerging.

\section{References}

1. Dawson C, Whitfield H. ABC of Urology. British Journal of Medicine 1996; 312: 961-4. 


\section{Reviews}

2. Manges AR, Johason JR. Widespread distribution of urinary tract infections caused by a multi resistant Escherichia coli clonal group. New England Journal of Medicine 2001; 345: 1007-13.

3. Akram M, Shahid M, Khan AU. Aetiology and antibiotic resistance patterns of community-acquired urinary tract infections in J N M C Hospital Aligarh, India Ann Clin Microbiol Antimicrob 2007; 23: 6-4.

4. Jamal W, Rotimi VO, Prevalence of extendedspectrum beta-lactamases in Enterobacteriaceae, Pseudomonas and Stenotrophomonas as determined by the VITEK 2 and E test systems in a Kuwait teaching hospital. Medical Principles and Practice 2005; 14(5): 325-31.

5. Bonadio M, Meini M, Current microbiological and clinical aspects of urinary tract infections. European Urology 2001; 40(4): 439-44.

6. Andreu A, Alós JI, Gobernado M, Marco F, Aetiology and antimicrobial susceptibility among uropathogens causing community-acquired lower urinary tract infection. Enferm Infecc Microbiol Clin. 2005; 23(1): 4-9. 